\title{
High Pulsed Magnetic Field Sensor Based on La-Ca-Mn-O Thin Polycrystalline Films
}

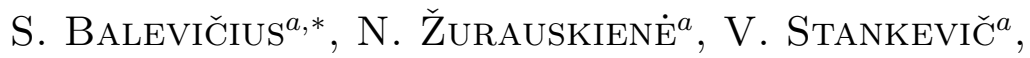 \\ S. Keršulis ${ }^{a}$, J. NoviCKiJ ${ }^{b}$, L.L. Altgilbers ${ }^{c}$ And F. Clarke $^{c}$ \\ ${ }^{a}$ Semiconductor Physics Institute, A. Goštauto 11, Vilnius, Lithuania \\ ${ }^{b}$ Vilnius Gediminas Technical University, Lithuania \\ ${ }^{c}$ US Army Space and Missile Defense Command, Huntsville AL, USA \\ It is demonstrated that polycrystalline $\mathrm{La}_{0.33} \mathrm{Ca}_{0.67} \mathrm{MnO}_{3}$ thin film sen- \\ sors can be used to measure pulsed strong magnetic fields with microsecond \\ duration rise and decay times. The response characteristics of these sen- \\ sors were investigated using $0.7-1.0 \mathrm{~ms}$ duration bell-shaped magnetic field \\ pulses of 10-20 T amplitudes and by using special waveform magnetic field \\ pulses with amplitudes of $40 \mathrm{~T}$ and decay times of $50 \mu \mathrm{s}$. The response of \\ these magnetic field sensors was compared with those of conventional loop \\ sensors and Faraday rotation sensors using $\mathrm{Bi}_{12} \mathrm{SiO}_{20}$ single crystals as a \\ known standard.
}

PACS numbers: 75.30.Kz, 73.40.Gk, 85.70.Ay, 61.72.Mm

\section{Introduction}

Sensors used in measuring high magnetic field pulses generated during magnetic flux compression, electromagnetic launching, or metal forming $[1,2]$ have to satisfy the following requirements: absence of saturation at high magnetic fields, high-speed responsiveness, and sensitivity independence to the direction of the magnetic field. The discovery of colossal magnetoresistance (MR) in manganites yields a new possibility in this area [3]. In the present paper, we report on the results of a study of $\mathrm{MR}$ in $\mathrm{La}_{0.33} \mathrm{Ca}_{0.67} \mathrm{MnO}_{3}$ (LCMO) films prepared by laser deposition on a polycrystalline substrate, demonstrating that high pulsed magnetic field sensors fabricated from these films are able to operate at microsecond times and that they have several advantages over loop sensors and sensors based on the Faraday rotation effect.

*corresponding author; e-mail: sbal@uj.pfi.lt 


\section{Experiment}

A polycrystalline $\mathrm{La}_{0.33} \mathrm{Ca}_{0.67} \mathrm{MnO}_{3}$ film was grown on a lucalox substrate using a pulsed deposition technique. The thickness $(d)$ of the films was about $400 \mathrm{~nm}$. The electrical resistivity $(R)$ dependence on temperature $(T)$ of the films had a flat maximum around $180 \mathrm{~K}$. The films exhibited a non-textured single-phase polycrystalline structure with $50 \mathrm{~nm}$ size grains. Electrical contacts, spaced by $20 \mu \mathrm{m}$ gaps, were made by thermal deposition of $\mathrm{Ag}$, using a Cr sub-layer. The $1 \mathrm{~mm}$ long and $1 \mathrm{~mm}$ wide sensors were soldered to bifilar twisted wires and covered by epoxy.

Testing of the sensors was performed in a pulsed magnetic field generator [3] using multi-shot magnetic field coils that produced sinusoidally shaped $1 \mathrm{~ms}$ duration magnetic pulses with amplitudes up to $45 \mathrm{~T}$. To produce pulses with $0.2 \mathrm{~ms}$ decay times, a special coil with rapidly "short-circuited" windings, was designed. The pulsed magnetic field generator was calibrated by using a conventional loop sensor and a Faraday rotation measurement system. The system (Fig. 1a) consisted of a

\section{(a)}
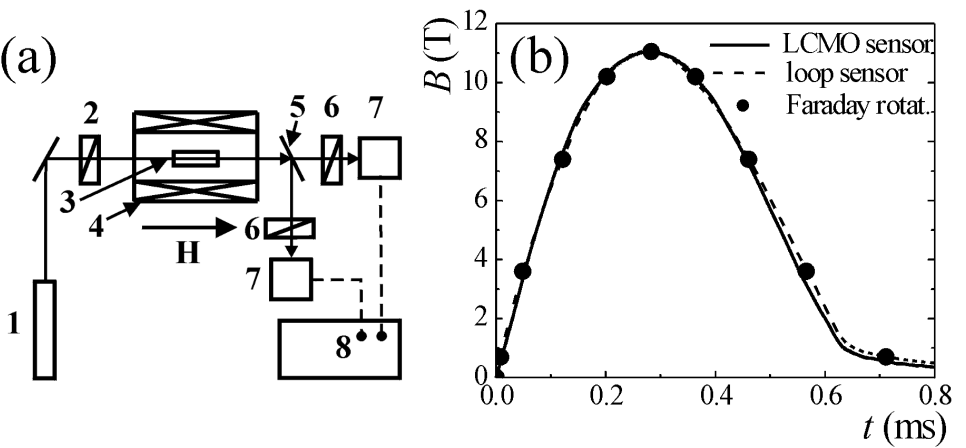

Fig. 1. (a) Schematic diagram of the experimental set-up for Faraday rotation measurements. (b) Pulsed magnetic field measurements using different sensors.

He-Ne laser - 1, polarizer — 2, magnetic field coil - 4, and two Glan-Foucault calcite prisms - 6. The light signal was detected by photodiode -7 , the voltage from which was recorded by oscilloscope - 8. The standard sample used in the Faraday rotation measurement was a $4 \mathrm{~mm}$ long $\mathrm{Bi}_{12} \mathrm{SiO}_{20}$ crystal - 3 . The dependence of the rotation of the polarization plane angle on magnetic field inductance $(B)$ (in tesla) was calculated from the formula: $B=\mu_{0} \theta / c l$, where $\theta$ is the angle of polarization plane rotation (in radians), $c$ is the Verdet constant ( $c=0.199$ for the $\mathrm{Bi}_{12} \mathrm{SiO}_{20}$ crystal at wavelength $\lambda=660 \mathrm{~nm}$ ), $l$ is the length of the crystal (in $\mathrm{cm}$ ), and $\mu_{0}$ is the vacuum magnetic permeability.

\section{Results and discussion}

A typical waveform of the magnetic field pulse measured with LCMO sensor based on $\mathrm{La}_{0.33} \mathrm{Ca}_{0.67} \mathrm{MnO}_{3}$ film is presented in Fig. $1 \mathrm{~b}$ (solid curve). The film 
was deposited using the first harmonic $\left(\lambda_{1}=1.06 \mu \mathrm{m}\right)$ of a YAG:Nd laser. The dashed curve shows measurement using a loop sensor, while the dots in Fig. 1b represent the Faraday rotation measurements. The magnetic field was applied in parallel to the direction of the bias current. Analogous waveforms of the pulse were obtained for other configurations of the field, substrate, and bias current. Figure 2a (curves 1) shows that sensors based on these films are able to record absolute values of the magnetic field, independent of the field orientation relative to the sensor's substrate plane and bias current direction. However, the effect of MR anisotropy is observed in the films grown by the second harmonic $\left(\lambda_{2}=0.53 \mu \mathrm{m}\right)$ of the laser (curves 2). This anisotropy is more pronounced when the applied magnetic field is parallel to the substrate plane and perpendicular to the bias current direction. The improvement of the film structure leads to the appearance of the stronger MR anisotropy effects in the film and is demonstrated by curves 3 in Fig. 2a obtained for epitaxial films grown on strontium titanate substrates.
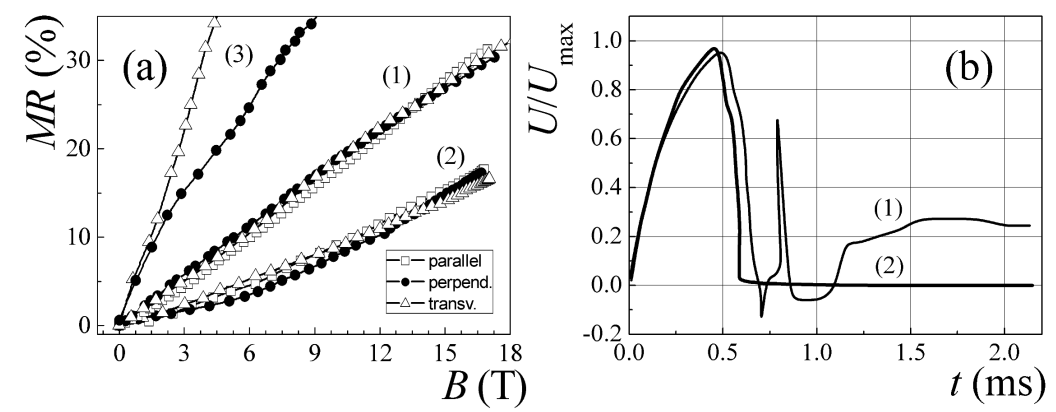

Fig. 2. (a) Magnetic field dependence of magnetoresistance $M R=[R(0)-R(B)] / R(0)$ for films grown by using the first (1) and second (2) harmonic of laser. The curves (3) obtained on epitaxial film grown on the $\mathrm{SrTiO}_{3}$ substrate. (b) Waveforms of responses of loop sensor (1) and sensor based on $\mathrm{La}_{0.33} \mathrm{Ca}_{0.67} \mathrm{MnO}_{3}$ (2) in "short-circuiting" of the coil's.

The response speed of the sensor was also evaluated using the "exploding" coils. Figure 2b shows time-dependent magnetic field measurements by a loop sensor (curve 1) and by a sensor based on polycrystalline manganite films (curve 2). Electric arcing and mechanical displacement of coil windings accompany the generation of abrupt drops in the magnetic field inside the coil. As can be seen in Fig. 2b, the loop sensor's response contains several sharp peaks generated as a result of the arcing process, which lasts for a certain period of time after the abrupt magnetic field drop. Meanwhile, the sensors, based on manganite film, recorded only the magnitude of the magnetic field inductance.

The results obtained can be explained by taking into consideration the polycrystalline structure of $\mathrm{La}_{0.33} \mathrm{Ca}_{0.67} \mathrm{MnO}_{3}$ film and properties of mesoscale regions surrounding the grain boundaries (GB) between crystallites [4]. The application 
of the magnetic field transforms such region from randomly oriented paramagnetic isolating state to magnetically ordered low resistance ferromagnetic state. As a result, the conductivity of the polycrystalline film is determined by the low resistivity ferromagnetic regions and by charge carrier tunnelling through the isolating GBs. When the magnetic field is switched off, the mesoscopic regions abruptly return back to their isolating paramagnetic state. This explains the fast reaction of the sensor to the rise and decay of the magnetic field. The non-perfect polycrystalline structure is also responsible for insensitivity of the sensor to magnetic field orientation. Films deposited by the first harmonic of the laser (Fig. 2a) contain a large number of imperfections because the evaporation in this case takes place deep in the target and small droplets of the substance propagate together with a molecular stream. However, films prepared by the second harmonic of the laser are textured and exhibit a small anisotropy of MR effect. The highest MR anisotropy was obtained on high quality epitaxial films (see curves 3 in Fig. 2a).

Finally, it should be concluded that in order to create fast high magnetic field sensors, which would be able to measure the absolute value of the magnetic inductance, it is necessary to have vitreous structures in the polycrystalline $\mathrm{La}_{0.33} \mathrm{Ca}_{0.67} \mathrm{MnO}_{3}$ film.

\section{Acknowledgments}

The work was supported by the Lithuanian Science and Studies Foundation contr. No. K-058.

\section{References}

[1] L.L. Altgilbers, Yu.V. Tkach, T. Yatsenko, Ya. Tkach, in: Proc. 14th IEEE Intern. Pulsed Power Conf., Dallas (Texas), Pulsed Power Conference, 2003, Vol. 2, U.S. Army Space Missile Defense Command, Huntsville (USA) 2003, p. 1085.

[2] V. Belyy, S.M. Fertik, L.T. Khimenko, Electromagnetic Metal Forming Handbook, Vischa Shkola, Kharkov State University, Kharkov 1977.

[3] J. Novickij, S. Balevičius, N. Žurauskienè, P. Cimmperman, L.L. Altgilbers, EPPS 2002, Saint Louis, IEE Proc., p. 30/1-30/6.

[4] Y.-A. Soh, G. Aeppli, N.D. Mathur, M.G. Blamire, Phys. Rev. B 63, 020402 (2000). 